\title{
Dynamic behaviour and stability analysis of a compensated aerostatic pad
}

\author{
Federico Colombo, Luigi Lentini, Terenziano Raparelli, Andrea Trivella,", \\ Vladimir Viktorov \\ Politecnico di Torino, C.so Duca degli Abruzzi 24, 10129, Torino, Italy
}

\begin{abstract}
Thanks to their low friction, aerostatic pads have important applications in precision positioning systems and linear guides. A simple and cheap solution to increase the static stiffness of aerostatic pads is to add a proper designed pneumatic valve to regulate the air flow supplied to the bearing. However, integrating aerostatic pads with additional devices can reduce its dynamic performance. This paper presents a numerical study on the dynamic behaviour and stability a commercial aerostatic pad controlled by a custom-built diaphragm valve. The bearing performance is studied by means of a lumped parameters model. Air bearing stiffness and damping are analysed in the frequency domain. Subsequently, the lumped model is linearized to investigate the stability of the system by means of RouthHurwitz method. The performance of the controlled air pad is compared to that of a simple commercial air pad.
\end{abstract}

\section{Introduction}

Aerostatic bearings are commonly used in applications that require high positioning accuracy. Due to their relative low stiffness, various solutions are used to improve the performance of these devices [1,2]. The use of multiple orifices [3-5], compound restrictors [6], microholes [7] and porous surfaces [8,9] as feeding system are possible solutions to increase the stiffness and load capacity of air pads. Moreover, stiffness can be further improved by using suitable active or passive compensation systems [10,11]. Active compensation systems can lead to significant performance improvement, but, due to the need for sensors, actuators and controllers, they represent an expensive solution. Conversely, despite their limited performance improvements, passive compensation systems are a cheaper alternative solutions than may be employed for industrial applications. Ghodsiyeh et al. [12] have proposed a passive compensation method consisting in the integration of an aerostatic pad and a diaphragm valve.

It was found numerically and experimentally that this kind of compensation makes it possible to obtain bearings with quasi-infinite and even negative static stiffness.

\footnotetext{
* Corresponding author: andrea.trivella@polito.it
} 
Experimental tests confirmed that a negative stiffness does not imply the presence of dynamic instability, but it is due to the overcompensation of the diaphragm valve. However, these analyses made not possible to define the stability threshold of the system and how the stability is related to the bearing design parameters.

This paper presents a numerical study of the dynamic behavior of the compensated pad using the lumped parameter model. Stiffness and damping are investigated using the perturbation method. Additionally, Routh-Hurwitz method is used to study stability of the linearized model of the pad. The performance of the here proposed system is compared to a commercial air pad used as a benchmark.

\section{Aerostatic pad description}

Figure 1a shows the geometry of the compensated air pad. The pad has a rectangular base ( $A=60 \mathrm{~mm}$ and $B=30 \mathrm{~mm}$ ) and four orifices of diameter $d=1 \mathrm{~mm}$. The orifices are located in the middle of the sides of a rectangular groove line of dimensions $a=45 \mathrm{~mm}$ and $b=20$ $\mathrm{mm}$. Figure $1 \mathrm{~b}$ shows the cross-section of the feeding hole. The groove presents a triangular cross-section of height $h_{g}=60 \mu \mathrm{m}$ and with $w_{g}=200 \mu \mathrm{m}$. The performance of the compensated pad is compared to that of a commercial pad of same shape, size and groove but with four holes of diameter $0.18 \mathrm{~mm}$. Moreover, the commercial pad has conical pockets downstream from each supply hole with a depth of $300 \mu \mathrm{m}$ and a diameter of $0.8 \mathrm{~mm}$. The reason of the different supply hole diameter is due to the presence of the regulating valve. To make the regulation more effective, it is convenient to reduce the pneumatic resistance of the supply holes of the compensated pad compared to that of the supply hole of the valve nozzle.

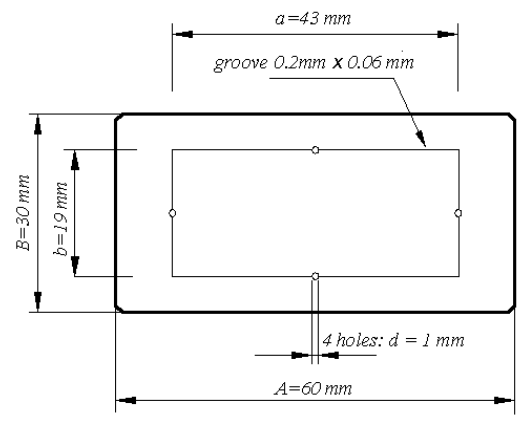

Fig. 1a. Compensated air pad.

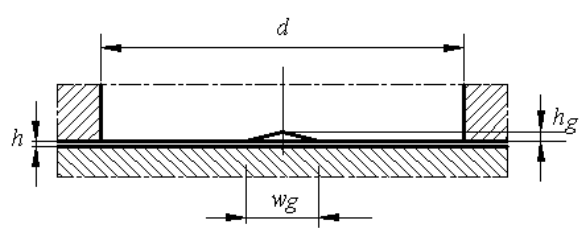

Fig. 1b. Supply hole cross geometry.

\section{Diaphragm valve}

Fig. 2 illustrates the scheme of the nozzle of the custom-built diaphragm valve. Compressed air is supplied to the valve at the absolute pressure $P_{S}$, passes through the nozzle (1) and it is exhausted from its diameter $d_{V}=0.5 \mathrm{~mm}$. The diaphragm (2) is a circular steel foil of diameter $D=6 \mathrm{~mm}$ and stiffness $k_{m}=180 \mathrm{~N} / \mathrm{mm}$. $P_{1}$ is the absolute pressure in the control chamber of the valve, $P_{a}$ is the ambient pressure. When $P_{1}=P_{a}$, the diaphragm is undeformed (dotted line) and the initial distance $x_{0}$ between the nozzle and the diaphragm can be manually regulated through a micrometric screw. On this regard, it was experienced 
that the compensating action of the valve is more efficient when, in the absence of air supply, the diaphragm is initially preloaded by the nozzle, i.e., for negative values of $x_{0}$.

The bending of the diaphragm preloaded by the nozzle causes the passage of a small air flow. The nozzle-diaphragm distance corresponding to the minimum flow rate passing through the valve has been identified with an equivalent distance $x_{\min }=12 \mu \mathrm{m}$.

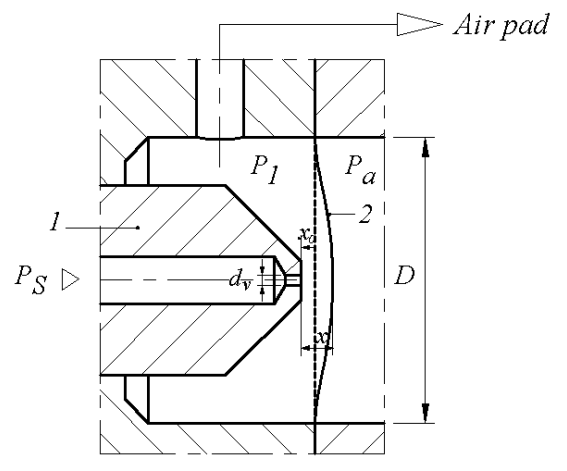

Fig. 2. Scheme of the control valve: enlargement of diaphragm and nozzle

\section{Mathematical lumped model}

The surface of the pad and the counter pad are assumed parallel and perfectly smooth. Figure 3 shows the pneumatic scheme of the system. It consists of a series of lumped pneumatic resistances and volumes. $R_{1}$ is the resistance of the nozzle-diaphragm, $R_{2}$ corresponds to the resistance of each supply hole of the pad, $R_{3}$ is the viscous resistance due to the air gap height $h$ of the pad. $V_{1}$ corresponds to the volumes of the supply duct of the pad, $V_{0}$ is the sum of the air gap volume $A B h$ and groove volumes $V_{g}\left(V_{0}=A B h+V_{g}\right)$.

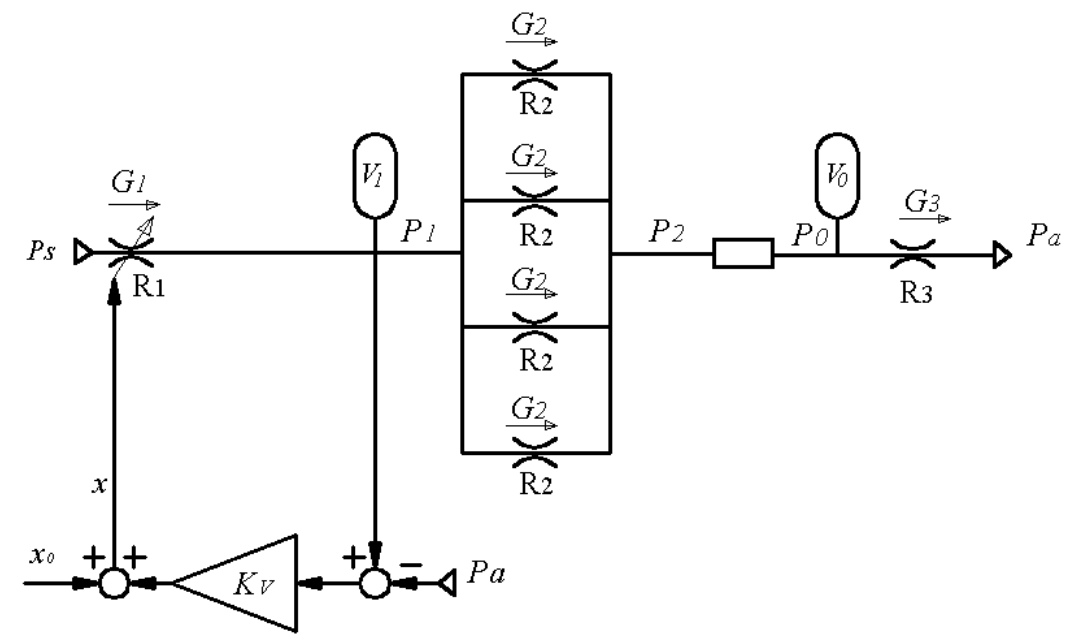

Fig. 3. Pneumatic scheme of the compensated air pad with the diaphragm pneumatic valve.

The distance $\mathrm{x}$ between nozzle and diaphragm is a linear function of the pressure $P_{1}$ : 


$$
x=x_{0}+k_{V}\left(P_{1}-P_{a}\right) ; k_{V}=\frac{\pi D^{2}}{4 k_{m}}
$$

$P_{2}$ is the absolute pressure immediately downstream each pad orifice. The mass flow through the first two resistances $R_{i}(i=1,2)$ is described by the ISO formula 6358 :

$$
G_{i}=\sqrt{T_{0} / T} C_{i} P_{i} \sqrt{1-\varphi_{i}^{2}} ; \varphi_{i}=\frac{\left(P_{d} / P_{u}\right)-b_{c}}{1-b_{c}}
$$

where $\varphi_{i}$ represents subsonic $\left(0<\varphi_{i} \leq 1\right)$ or sonic $\left(\varphi_{i}=0\right)$ conditions, $P_{u}, P_{d}$ are the upstream and downstream absolute pressures of each resistance, $b_{c}$ is the pressure critical ratio, assumed equal to $0.528 . T_{0}$ and $T$ are a reference $\left(T_{0}=293 \mathrm{~K}\right)$ and the environmental air temperatures. $C_{i}$ is the conductance of the ith cross-section. The expression of $C_{2}$ takes into account the presence of the grooves crossing the pockets:

$$
C_{1}=\psi c_{d_{a, 1}} \pi d_{V} x ; C_{2}=\psi c_{d_{a, 2}}\left(\pi d h+w_{g} h_{g}\right)
$$

where $\psi=0.686 / \sqrt{R T}, \mathrm{R}=287 \mathrm{~J} \mathrm{~kg}-1 \mathrm{~K}$, and $c_{d a, i}(\mathrm{i}=1,2)$ are the discharge coefficients at the nozzle and pad holes outlet, which were experimentally obtained in [13]:

$$
\begin{gathered}
c_{d a, i}=1.05\left(1-0.3 \mathrm{e}^{-0.005 R e_{i}}\right) \\
R e_{1}=\frac{G_{1}}{\pi \mu d_{V}} ; R e_{2}=\frac{G_{2} h}{\pi \mu d h_{e q}} ; h_{e q}=\frac{\pi d h+w_{g} h_{g}}{\pi d}
\end{gathered}
$$

where $R e_{i}$ is the Reynolds number. The pressure distribution was computed on the basis of the 2D Reynolds equation under isothermal conditions:

$$
\frac{d P}{d x}+12 \mu R T \frac{g_{x}}{P h^{3}}=0 ; \frac{d P}{d y}+12 \mu R T \frac{g_{y}}{P h^{3}}=0
$$

where $g_{x}$ and $g_{y}$ are the mass flow rates per unit of width along the $x$ and $y$ directions and $\mu$ is the air dynamic viscosity. The mass flow rates $G_{x}$ and $G_{y}$, outgoing from each side of the rectangular groove are obtained by integration of the Reynolds equations. The total air consumption $G$ of the pad is obtained as follows:

$$
\begin{gathered}
G=G_{3}=2\left(G_{x}+G_{y}\right)=\frac{1}{6 \mu R T} v\left(P_{0}^{2}-P_{a}^{2}\right) h^{3} ; \\
v=\left(\frac{b}{A-a}+\frac{a}{B-b}\right)
\end{gathered}
$$

The model assumes a constant pressure $P_{0}$ under the rectangular area surrounded by the groove, whereas, outside this area, it decreases linearly up to the ambient pressure $P_{a}$. The pressure $P_{0}$ is identified as a function of $P_{2}$ and $h$, where $h$ is expressed in $[\mu m]$ :

$$
P_{0}=f\left(P_{2}-P_{a}\right)+P_{a}=\left(1-c_{1}\left(\frac{c_{2}}{h}\right)\right)\left(P_{2}-P_{a}\right)+P_{a}
$$

A good identification of $P_{0}$ is obtained with $c_{1}=0.14 ; c_{2}=5 \mu \mathrm{m}$. Integrating the pressure distribution under the pad, the correspondent pressure force $F_{p}$ results equal to: 


$$
F_{p}=S_{e q}\left(P_{0}-P_{a}\right) ; S_{e q}=\frac{1}{3}\left[a b+A B+\frac{(A b+a B)}{2}\right]
$$

The dynamic behavior of the pad is studied by linearizing the equations of the model through the perturbation method. The expressions of the mass flow rates, continuity equations and Equation (4) are linearized around a neighbour of a static equilibrium position of the air pad and their variations are expressed in Laplace domain. Also, equations (1), (8) are expressed in Laplace domain.

$$
\begin{aligned}
& \overline{G_{1}}=k_{1} \bar{x}+k_{2} \overline{P_{1}} ; \overline{G_{2}}=k_{3} \overline{P_{1}}+k_{4} \overline{P_{2}}+k_{5} \bar{h} ; \\
& \overline{G_{3}}=k_{6} \overline{P_{0}}+k_{7} \bar{h} ; \overline{G_{1}}-4 \overline{G_{2}}=k_{8} s \overline{P_{1}} ; \\
& 4 \overline{G_{2}}-\overline{G_{3}}=k_{9} s \bar{h}+k_{10} s \overline{P_{0}} ; \\
& \overline{P_{0}}=k_{11} \overline{P_{2}}+k_{12} \bar{h} ; \\
& \bar{x}=k_{13} \overline{P_{1}} ; \overline{F_{p}}=S_{e q} \overline{P_{0}}
\end{aligned}
$$

where:

$$
\begin{aligned}
& k_{1}=\left.\frac{\partial G_{1}}{\partial x}\right|_{0}=\psi \pi d_{V} c_{d a, 1} P_{S} \sqrt{1-\varphi_{1}^{2}} ; \varphi_{1}=0 \text { if } \frac{P_{1}}{P_{S}} \leq b_{c} \\
& k_{1}=\left.\frac{\partial G_{1}}{\partial x}\right|_{0}=0 \text { if } x<x_{\text {min }} ; \\
& k_{2}=\left.\frac{\partial G_{1}}{\partial P_{1}}\right|_{0}=-\frac{C_{1}}{1-b_{c}} \frac{\varphi_{1}}{\sqrt{1-\varphi_{1}^{2}}} ; \\
& k_{3}=\left.\frac{\partial G_{2}}{\partial P_{1}}\right|_{0}=C_{2}\left(\sqrt{1-\varphi_{2}^{2}}+\frac{P_{2}}{P_{1}} \frac{1}{1-b} \frac{\varphi_{2}}{\sqrt{1-\varphi_{2}^{2}}}\right) ; \varphi_{2}=0 \quad \text { if } \frac{P_{2}}{P_{1}} \leq b_{c} \\
& k_{4}=\left.\frac{\partial G_{2}}{\partial P_{2}}\right|_{0}=-\frac{C_{2}}{1-b_{c}} \frac{\varphi_{2}}{\sqrt{1-\varphi_{2}^{2}}} k_{5}=\left.\frac{\partial G_{2}}{\partial h}\right|_{0}=\psi \pi d c_{d a, 2} P_{1} \sqrt{1-\varphi_{2}^{2}} ; \\
& k_{6}=\left.\frac{\partial G_{3}}{\partial P_{0}}\right|_{0}=\frac{P_{0}}{3 \mu R T} v h^{3} ; \\
& k_{7}=\left.\frac{\partial G_{3}}{\partial h}\right|_{0}=\frac{v h^{2}}{6 \mu R T}\left[2 P_{0}\left(P_{2}-P_{a}\right) \frac{d f}{d h} h+3\left(P_{0}{ }^{2}-P_{a}^{2}\right)\right] ; \\
& k_{8}=\frac{V_{1}}{R T} ; k_{9}=\frac{P_{0} A B}{R T} ; k_{10}=\frac{V_{0}}{R T} ; k_{11}=f ; \\
& k_{12}=\left(P_{2}-P_{a}\right) \frac{d f}{d h} ; \frac{d f}{d h}=\frac{c_{2}}{h^{2}} \cdot c_{1} \frac{c_{2}}{h} \cdot \ln c_{1} ; k_{13}=k_{V}
\end{aligned}
$$

It is worth noting that, the exponential term of $c_{d a, 2}$ has been considered constant in computing the partial derivative of $G_{2}$ with respect to h. Making use of equations (9), it is possible to compute the transfer function $\overline{\mathrm{F}_{\mathrm{P}}} / \overline{\mathrm{h}}$ :

$H(s)=\frac{\overline{F_{P}}}{\bar{h}}=\frac{a_{0}}{b_{0}} S_{e q} \frac{1+\left(a_{1} / a_{0}\right) s+\left(a_{2} / a_{0}\right) s^{2}}{1+\left(b_{1} / b_{0}\right) s+\left(b_{2} / b_{0}\right) s^{2}}=K_{S} \frac{1+\tau_{1} s+\tau_{2} s^{2}}{1+\gamma_{1} s+\gamma_{2} s^{2}}$

where: 


$$
\begin{aligned}
& a_{0}=16\left(k_{3} k_{4} k_{12}-k_{3} k_{5} k_{11}\right)-k_{11}\left(k_{1} k_{13}+k_{2}-4 k_{3}\right)\left(4 k_{5}-k_{7}-\frac{4 k_{4} k_{12}}{k_{11}}\right) ; \\
& a_{1}=k_{9} k_{11}\left(k_{1} k_{13}+k_{2}-4 k_{3}\right)+k_{8} k_{11}\left(4 k_{5}-k_{7}-\frac{4 k_{4} k_{12}}{k_{11}}\right) ; \\
& a_{2}=-\mathrm{k}_{8} \mathrm{k}_{9} \mathrm{k}_{11} ; \\
& b_{0}=16 \mathrm{k}_{3} \mathrm{k}_{4}-\mathrm{k}_{11}\left(\mathrm{k}_{1} \mathrm{k}_{13}+\mathrm{k}_{2}-4 \mathrm{k}_{3}\right)\left(\mathrm{k}_{6}-\frac{4 \mathrm{k}_{4}}{\mathrm{k}_{11}}\right) ; \\
& b_{1}=k_{8} k_{11}\left(k_{6}-\frac{4 k_{4}}{k_{11}}\right)-k_{10} k_{11}\left(k_{1} k_{13}+k_{2}-4 k_{3}\right) ; \\
& b_{2}=k_{8} k_{10} k_{11} ;
\end{aligned}
$$

$K_{S}=\frac{a_{0}}{b_{0}} S_{e q}$ is the static stiffness of the air pad. By expressing the transfer function $H(s)$ in the frequency domain, the theoretical dynamic stiffness $K$ and damping $c$ of the air under the pad are computed as:

$$
\begin{gathered}
K(\omega)=-\operatorname{Re}_{H}(\omega)=-K_{S} \frac{1+\left(\tau_{1} \gamma_{1}-\tau_{2}-\gamma_{2}\right) \omega^{2}+\tau_{2} \gamma_{2} \omega^{4}}{\left(1-\gamma_{2} \omega^{2}\right)^{2}+\left(\gamma_{1} \omega\right)^{2}} \\
c(\omega)=-\frac{\operatorname{Im}_{H}(\omega)}{\omega}=-K_{S} \frac{\left(\tau_{1}-\gamma_{1}\right)+\left(\tau_{2} \gamma_{1}-\tau_{1} \gamma_{2}\right) \omega^{2}}{\left(1-\gamma_{2} \omega^{2}\right)^{2}+\left(\gamma_{1} \omega\right)^{2}}
\end{gathered}
$$

The equilibrium equation of the pad is:

$$
\bar{F}-\overline{F_{p}}+M s^{2} \bar{h}=0
$$

where $\mathrm{F}$ is the vertical external load applied to the pad and $\mathrm{M}$ the mass related to the supported payload $(F=M g)$. By defining $G(s)=-\frac{1}{M s^{2}}$, it is possible to obtain the closed loop block diagram of Figure 4 . The equivalent transfer function of the entire system is:

$$
\begin{aligned}
& G_{e q}(s)=\frac{G(s)}{1+G(s) H(s)}=\frac{1}{K_{S}} \frac{\left(1+\gamma_{1} s+\gamma_{2} s^{2}\right)}{\left(1+\delta_{1} s+\delta_{2} s^{2}+\delta_{3} s^{3}+\delta_{4} s^{4}\right)}=\frac{\bar{h}}{\bar{F}} \\
& \delta_{1}=\tau_{1} ; \delta_{2}=\tau_{2}-\frac{M}{K_{S}} ; \delta_{3}=-\frac{M \gamma_{1}}{K_{S}} ; \delta_{4}=-\frac{M \gamma_{2}}{K_{S}}
\end{aligned}
$$

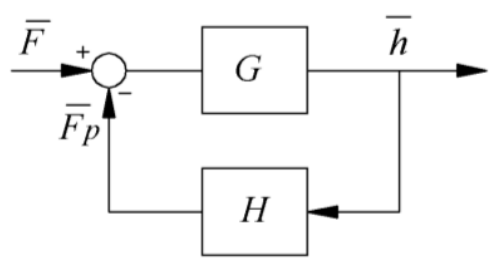

Fig. 4. Scheme of the feedback system. 


\section{Numerical results}

\subsection{Static analysis}

The static load capacities of the compensated air pad with different parameters $\mathrm{x} 0, \mathrm{dv}, \mathrm{d}, \mathrm{km}$ are analysed. Table 1 shows the selected parameters in few different operative cases. Figure 5a shows the load capacity versus the air gap height. The comparison of the results for the compensated and the commercial air pads is made with the same supply pressure $P_{S}=0.5$ $\mathrm{MPa}$.

Table 1. Operative cases for compensated and commercial air pad

\begin{tabular}{|c|c|c|c|c|c|}
\hline & & $\mathrm{x}_{0}(\mu \mathrm{m})$ & $\mathrm{d}_{\mathrm{v}}(\mathrm{mm})$ & $\mathrm{d}(\mathrm{mm})$ & $\mathrm{km}_{\mathrm{m}}(\mathrm{N} / \mathrm{mm})$ \\
\hline \multirow{5}{*}{ Compensated air pad } & case a & -20 & 0.5 & 1 & 180 \\
\hline & case b & -20 & 0.5 & 0.18 & 180 \\
\hline & case c & -20 & 0.5 & 0.18 & 270 \\
\hline & case d & -20 & 0.5 & 1 & 270 \\
\hline & case e & -30 & 0.5 & 1 & 180 \\
\hline Commercial air pad & case $\mathrm{f}$ & - & - & 0.18 & - \\
\hline
\end{tabular}

Compared to the commercial air pad, the controlled one globally presents a lower load capacity but it exhibits a significantly higher stiffness. Moreover, as discussed in [12], due to the regulating action of the valve, the characteristic curves of the compensated pad can be divided in three different regions (Fig. 5b): a by-pass (A-B), compensation (B-C) and a saturation zone (C-D). When the system is lightly loaded (by-pass), the distance between the nozzle and the diaphragm is quite small and compensated pad behaves has a lumped pneumatic resistance $\left(x=x_{\min }=x_{b y-p a s s}\right)$. As the load increases, the pressure in the valve chamber increases, the valves start regulating when $x>x_{b y-p a s s}$ (compensation zone).

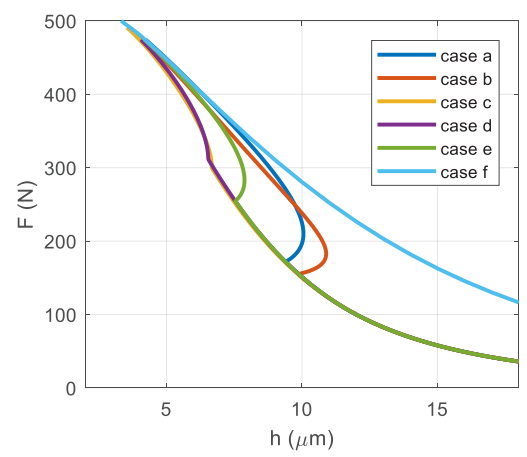

(a)

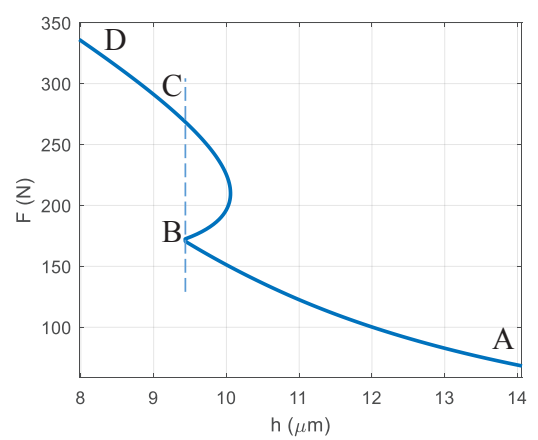

(b)

Fig. 5. a) Static load vs. air gap height, Ps $=0.5 \mathrm{MPa}$, commercial and compensated pads; b) A-B: bypass zone, B-C: compensation zone, C-D: saturation zone.

Here, the compensating action of the valve makes it possible to compensate the air gap variations due to the change of the external load by increasing the air flow supplied to the pad. Beyond point $C\left(h_{C}=h_{B}\right)$ the compensation is no more able to provide a suitable amount of air flow to compensate for air gap height variations. As can be seen, given the pad features, the characteristic shape of the load capacity curve is significantly influenced by the design parameters of the valve $\left(x_{0}, d_{v}\right.$, and $\left.k_{m}\right)$. On this regard, Colombo et al. proposed a design 
methodology to select the values of $x_{0}, k_{m}$ that maximize the stiffness of the system at a desired air gap height [14]. Curve a) of figure 5 corresponds to the design condition of the control system.
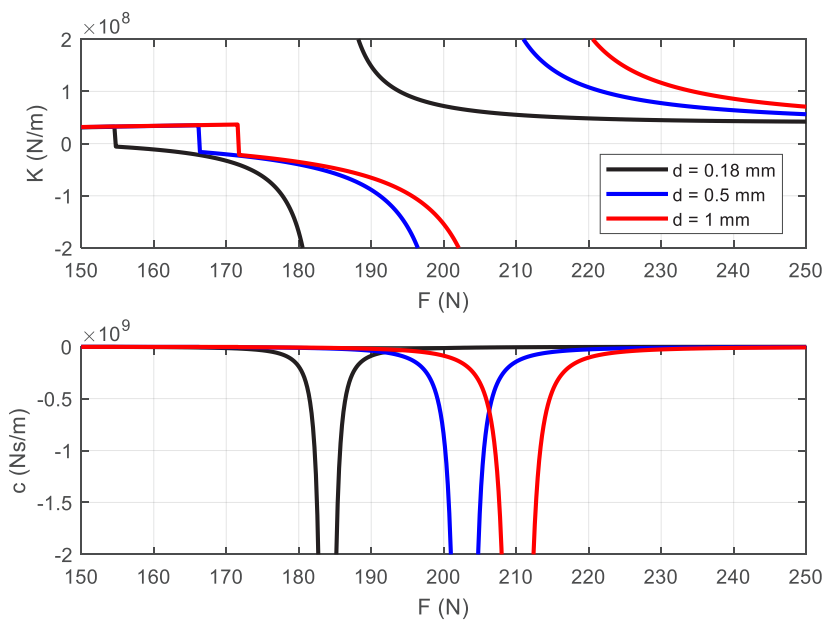

Fig. 6. Stiffness and damping of the compensated pad in static conditions, for some diameters $d$ : $P_{S}=0.5 \mathrm{MPa}, x_{0}=-20 \mu \mathrm{m}, d_{v}=0.5 \mathrm{~mm}, k_{m}=1.810^{5} \mathrm{~N} / \mathrm{m}$.

Figure 6 shows the trends of the air pad stiffness $K$ and the damping coefficient c obtained from equation (12) in static conditions $(\omega=0)$. The trends of the static stiffness expressed as functions of the external payload $F$ are reported by considering different sizes of the supply hole diameters of the compensated pad $(\mathrm{d}=0.18,0.5,1 \mathrm{~mm})$. It can be seen that, the stiffness changes sign in the regulation zone and it locally assumes values that tend to infinity, even $\mathrm{c}$ tends to infinity when $K_{S}$ tends to infinity.

\subsection{Dynamic analysis}

The analysis of the dynamic behaviour is aimed at investigating the stiffness and damping values of the air gap as the frequency $\omega$ of the applied load $\mathrm{F}$ varies. The results are obtained with the design parameters $\left(P_{S}=0.5 \mathrm{MPa}, x_{0}=-20 \mu \mathrm{m}, d_{v}=0.5 \mathrm{~mm}, \mathrm{~d}=1 \mathrm{~mm}, k_{m}=1.8\right.$ $10^{5} \mathrm{~N} / \mathrm{m}, V_{1}=1.410^{-6} \mathrm{~m}^{3}$ ), moreover the investigations regard the air gap height values belonging to the compensation region. Figure 7 shows the trends of the dynamic stiffness and damping in the presence of different air gap heights within the regulation zone. Figure $7 \mathrm{a}$ shows the results for frequencies till to $100 \mathrm{~Hz}$, figure $7 \mathrm{~b}$ shows better the same results in the range of frequency from $10 \mathrm{~Hz}$ to $50 \mathrm{~Hz}$. These results can be compared to those of figure 8 where the frequency response of the commercial pad is shown [15]. As can be seen, the stiffness and damping of the compensated pad reduce with the excitation frequency. In particular, both stiffness and damping drastically reduces even at low frequencies: at about 2 $\mathrm{Hz}$, the stiffness halves and the damping reduces by 5 times. Meanwhile, the stiffness and damping of the commercial pad present two opposite trends with respect to the excitation frequency. The stiffness increases, whereas the damping reduces. Figure 9 shows the effect of the volume $V_{1}$ of the connecting ducts between valve and pad. Here, it is possible to see that increasing the volume $V_{1}$ makes reduces the compensating action of the valve. 

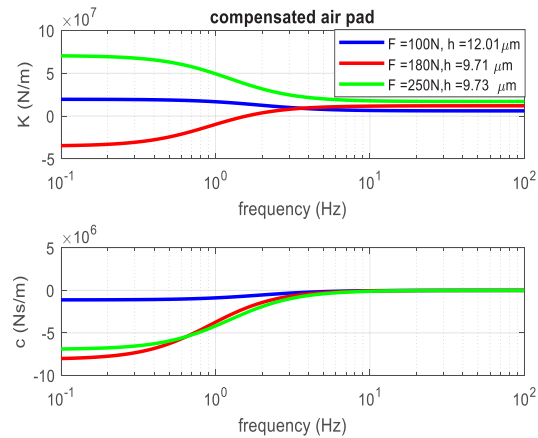

Fig. 7a. Compensated pad: dynamic stiffness and damping vs. frequency (from $0.1 \mathrm{~Hz}$ to 100 $\mathrm{Hz}) . P_{S}=0.5 \mathrm{MPa}, x_{0}=-20 \mu \mathrm{m}, d_{v}=0.5 \mathrm{~mm}$, $k_{m}=1.810^{5} \mathrm{~N} / \mathrm{m}, V_{1}=1.4 \cdot 10^{-6} \mathrm{~m}^{3}$
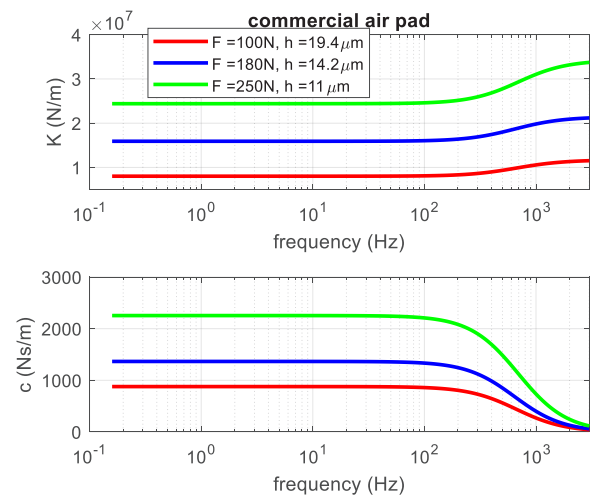

Fig. 8. Dynamic stiffness and damping vs. frequency for commercial pad, $P_{S}=0.5 \mathrm{MPa}, d$ $=0.18 \mathrm{~mm}$
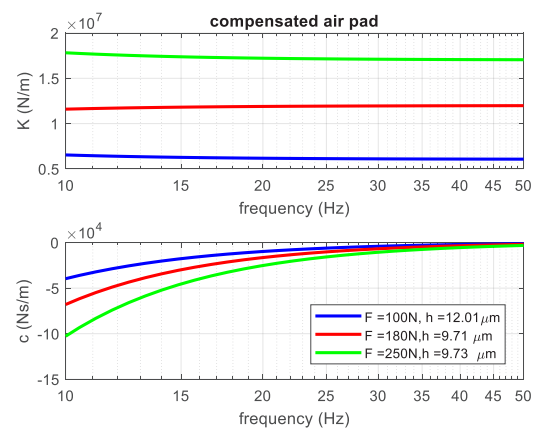

Fig. 7b. Compensated pad: dynamic stiffness and damping vs. frequency (from $10 \mathrm{~Hz}$ to 50 $\mathrm{Hz}), P_{S}=0.5 \mathrm{MPa}, x_{0}=-20 \mu \mathrm{m}, d_{v}=0.5$ $\mathrm{mm}, k_{m}=1.810^{5} \mathrm{~N} / \mathrm{m}, V_{1}=1.4 \cdot 10^{-6} \mathrm{~m}^{3}$
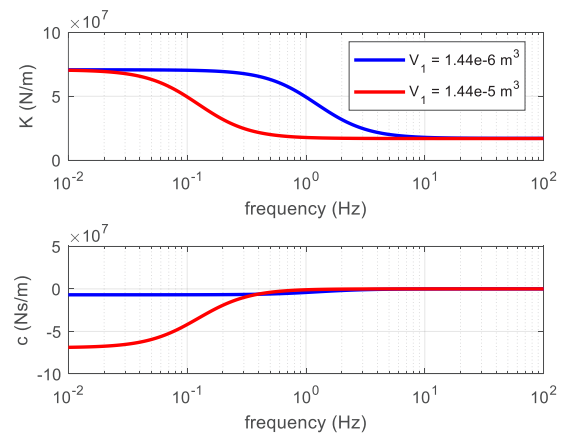

Fig. 9. Compensated pad. Dynamic stiffness and damping vs. frequency, effect of the volume $V_{1} . P_{S}=0.5 \mathrm{MPa}, x_{0}=-20 \mu \mathrm{m}, d_{v}$ $=0.5 \mathrm{~mm}, d=1 \mathrm{~mm}, k_{m}=1.810^{5} \mathrm{~N} / \mathrm{m}, F=$ $250 \mathrm{~N}, h=9.73 \mu \mathrm{m}$

\subsection{Stability analysis}

The dynamic results shown are related to the design operative condition of the pad. Both experimentation and simulations confirm stable operation in this situation. However, the controlled pad may not be stable in other working conditions. The stability is then theoretically analyzed by applying the Routh-Hurwitz criterion to the transfer function $G_{e q}$. The stability of the controlled air pad requires that all the coefficients $\delta_{1}, \delta_{2}, \delta_{3}, \delta_{4}$ must be positive. Moreover, the following two conditions (15) must be also satisfied:

$$
f_{1}=\delta_{2}-\frac{\delta_{1} \delta_{4}}{\delta_{3}}>0 ; f_{2}=\delta_{1}-\frac{\delta_{3}}{f_{1}}>0
$$

The stability of the system is evaluated by modifying the design parameter of the system $\left(P_{S}, x_{0}, d_{v}, d, k_{m}, V_{1}\right)$ with respect to a stable operation selected as reference (case a of Figure 5). According to the Routh-Hurwitz method, the stability is evaluated by considering the signs of the coefficients $\delta_{1}, \delta_{2}, \delta_{3}, \delta_{4}$ and the functions $f_{1}, f_{2}$. In all the examined cases, the coefficients $\delta_{1}, \delta_{2}, \delta_{3}, \delta_{4}$ and the function $f_{1}$ are always positive while the function $f_{2}$ 
can assume negative values. Figures 10-14 show the fields of instability (gray areas) identified in this study. The stability maps are reported as functions of the load capacity. The range of load within which the behavior is observed includes the zone of normal operation of the air pad. It was found that, the system can become unstable in several cases: as the distance $x_{0}$ increases (or as $d_{v}$ increases), as $d$ increases, as $k_{m}$ decreases, as $V_{1}$ decreases, as $P_{S}$ increases. The unstable operating zones enlarge when the variation of the parameters increases with respect to the values relating to a stable operating condition.

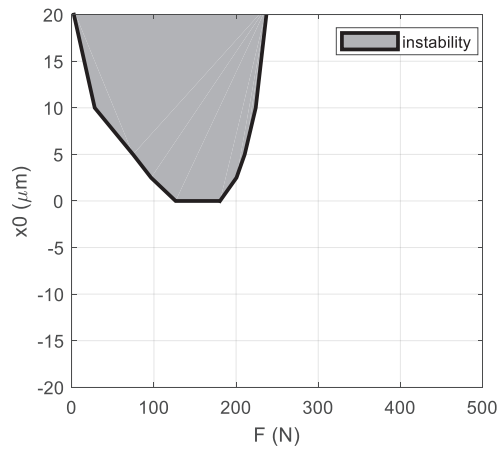

Fig. 10. Stability map as $x_{0}$ varies: $P_{S}=0.5$ $\mathrm{MPa}, d_{v}=0.5 \mathrm{~mm}, d=1 \mathrm{~mm}, k_{m}=1.810^{5}$ $\mathrm{N} / \mathrm{m}, V_{1}=1.410^{-6} \mathrm{~m}^{3}$

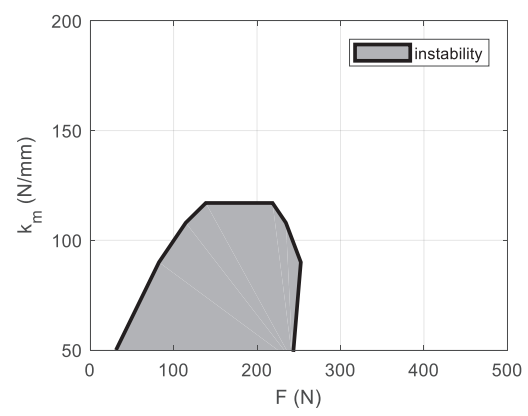

Fig. 12. Stability map as $k_{m}$ varies: $P_{S}=$ $0.5 \mathrm{MPa}, x_{0}=-20 \mu \mathrm{m}, d_{v}=0.5 \mathrm{~mm}, d=$ $1 \mathrm{~mm}, V_{1}=1.410^{-6} \mathrm{~m}^{3}$

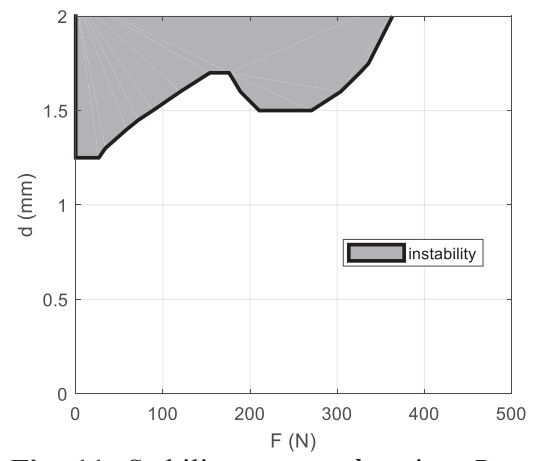

Fig. 11: Stability map as $d$ varies: $P_{S}=0.5$ $\mathrm{MPa}, x_{0}=-20 \mu \mathrm{m}, d_{v}=0.5 \mathrm{~mm}, k_{m}=1.8$ $10^{5} \mathrm{~N} / \mathrm{m}, V_{1}=1.410^{-6} \mathrm{~m}^{3}$

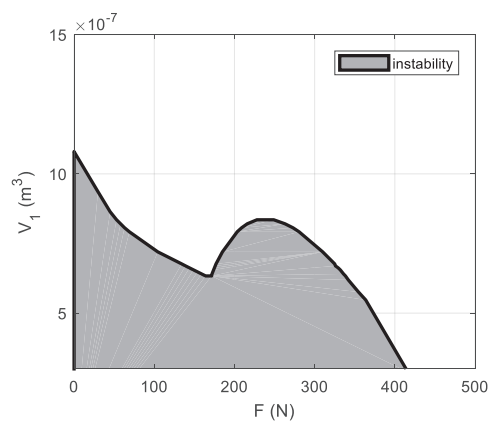

Fig. 13. Stability map as $V_{1}$ varies: $P_{S}=0.5$ $\mathrm{MPa}, x_{0}=-20 \mu \mathrm{m}, d_{v}=0.5 \mathrm{~mm}, d=1 \mathrm{~mm}$, $k_{m}=1.810^{5} \mathrm{~N} / \mathrm{m}$.

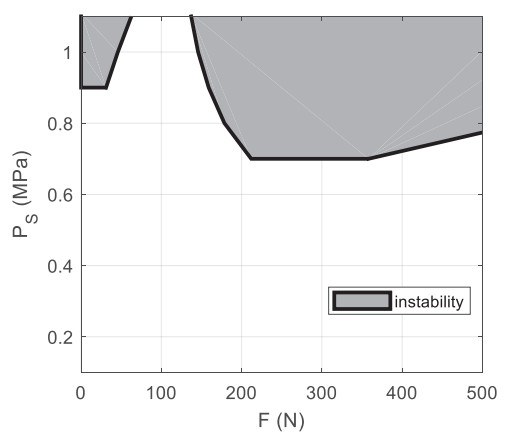

Fig. 14. Stability map as $P_{S}$ varies: $x_{0}=-20 \mu \mathrm{m}, d_{v}=0.5 \mathrm{~mm}, d=1 \mathrm{~mm}, k_{m}=1.810^{5} \mathrm{~N} / \mathrm{m}$, $V_{1}=1.410^{-6} \mathrm{~m}^{3}$ 


\section{Conclusion}

The present paper presented a numerical analysis of the dynamic behavior and stability of an aerostatic pad controlled by a diaphragm valve. The numerical model has been linearized to investigate the dynamic stiffness and damping of the compensated air pad. Within the regulating zone the static stiffness is significantly greater than that of the commercial air pad. Damping and stiffness decrease as the load application frequency increases and if the static operating point is very close to the point of infinite stiffness the dynamic stiffness is even greater than that of the commercial air pad. However, as the frequency varies, the damping remains much higher than that of the commercial pad.

The linearization of the model has made it possible to evaluate the stability of the system through the Routh-Hurwitz criterion. It has been found that the chosen design parameters allow a stable operation; the system is more prone to the instability at lower loads (higher air gap heights). However also the variation of the pad design parameters may lead to instability. In particular, the system can be unstable also at higher loads if the supply pressure increases. In fact, as the supply pressure increases, the instability field expands rapidly in almost the entire range of air gap heights. Moreover, it has been found that the system stability can be improved by increasing the volume of the ducts connecting the air pad to the valve, nevertheless, this also produces a significant reduction of the dynamic stiffness and damping. Ultimately, compared to the commercial air pad, the compensated one exhibit significantly higher performance for quasi-static applications.

Compared to other stability methods, Routh-Hurwitz criterion can be easily employed to verify the stability of dynamical systems and obtaining stability maps by considering different operating parameters of the investigated system. However, this method does not provide any information about the stability margin of the system. For this reason, feature works will be devoted to integrate the information related to the stability maps obtained through Routh-Hurwitz criterion with information regarding the stability margin of the investigated system, e.g., Nyqvist criterion. Moreover, these theoretical results will be verified through experiments.

\section{References}

1. Q. Gao, W. Chen, L. Lu, D. Huo, and K. Cheng, Tribology International (2019)

2. L. Lentini, M. Moradi, and F. Colombo, Tribology in Industry 40, 165 (2018).

3. 3. D. A. Boffey, A. E. Duncan, and J. K. Dearden, Tribology International 14, 287 (1981).

4. S. Z. Kassab, E. M. Noureldeen, and M. A. Shawky, Tribology International 30, 533 (1997).

5. F. Colombo, L. Lentini, T. Raparelli, A. Trivella, and V. Viktorov, Tribology Letters 66, (2018).

6. T. Nakamura and S. Yoshimoto, Tribology International 29, 145 (1996).

7. A. Charki, K. Diop, S. Champmartin, and A. Ambari, International Journal of Mechanical Sciences 72, 28 (2013).

8. M. Fourka and M. Bonis, Wear 210, 311 (1997).

9. T. S. Luong, W. Potze, J. B. Post, R. A. J. Van Ostayen, and A. Van Beek, Tribology International 37, 825 (2004).

10. F. Al-Bender, Precision Engineering 33, 117 (2009).

11. T. Raparelli, V. Viktorov, F. Colombo, and L. Lentini, Precision Engineering 44, 1 (2016).

12. D. Ghodsiyeh, F. Colombo, L. Lentini, T. Raparelli, A. Trivella, and V. Viktorov, Tribology International 141, (2020). 
13. G. Belforte, T. Raparelli, V. Viktorov, and A. Trivella, Tribology International 40, 512 (2007).

14. F. Colombo, L. Lentini, T. Raparelli, A. Trivella, and V. Viktorov, Lubricants 9, 47 (2021).

15. F. Colombo, L. Lentini, T. Raparelli, A. Trivella, and V. Viktorov, in International Conference on Robotics in Alpe-Adria Danube Region (Springer, 2018), pp. 678686. 